\title{
Erratum to: Development and modelling of different decarbonization scenarios of the European energy system until 2050 as a contribution to achieving the ambitious $1.5^{\circ} \mathrm{C}$ climate target-establishment of open source/data modelling in the European H2020 project openENTRANCE
}

H. Auer(D, P. Crespo del Granado, P.-Y. Oei, K. Hainsch, K. Löffler, T. Burandt, D. Huppmann, I. Grabaak

Published online March 29, 2021

(C) Springer-Verlag GmbH Austria, ein Teil von Springer Nature 2021

Erratum to: E\&l, Elektrotech. Inf.tech. 137(7) (2020) 346-358 https://doi.org/10.1007/s00502-020-00832-7

The following acknowledgment is missing in the original article:

\section{Acknowledgements}

This project has received funding from the European Union's Horizon 2020 Research and Innovation Programme under Grant Agreement No. 835896.

Publisher's Note Springer Nature remains neutral with regard to jurisdictional claims in published maps and institutional affiliations.
The original article can be found online at https://doi.org/10.1007/s00502-020-00832-7

Auer, Hans, Technische Universität Wien (TU-Wien) - Energy Economics Group (EEG), Gusshausstraße 25-29/E370-03, 1040 Vienna, Austria (E-mail: auer@eeg.tuwien.ac.at); Crespo del Granado, Pedro, Norwegian University of Science and Technology (NTNU), Trondheim, Norway; Oei, Pao-Yu, Technische Universität Berlin (TU-Berlin), Berlin, Germany; Hainsch, Karlo, Technische Universität Berlin (TU-Berlin), Berlin, Germany; Löffler, Konstantin, Technische Universität Berlin (TU-Berlin), Berlin, Germany; Burandt, Thorsten, Technische Universität Berlin (TU-Berlin), Berlin, Germany; Huppmann, Daniel, International Institute for Applied Systems Analysis (IIASA), Laxenburg, Austria; Grabaak, Ingeborg, Sintef, Trondheim, Norway 